A highly sensitive YBCO serial SQUID magnetometer with a flux focuser

This article has been downloaded from IOPscience. Please scroll down to see the full text article.

2006 Supercond. Sci. Technol. 19 S246

(http://iopscience.iop.org/0953-2048/19/5/S17)

The Table of Contents and more related content is available

Download details:

IP Address: 140.112.113.225

The article was downloaded on 24/07/2009 at 06:40

Please note that terms and conditions apply. 


\title{
A highly sensitive YBCO serial SQUID magnetometer with a flux focuser
}

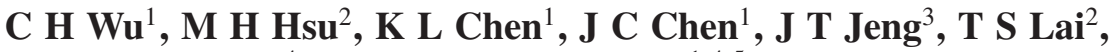 \\ Herng-Er Horng ${ }^{4}$ and Hong-Chang Yang ${ }^{1,4,5}$ \\ ${ }^{1}$ Department of Physics, National Taiwan University, Taipei 10617, Taiwan \\ ${ }^{2}$ Department of Physics, Chung Yuan Christian University, Chung Li 320, Taiwan \\ ${ }^{3}$ Institute of Mechatronic Engineering, National Taipei University of Technology, Taipei 106, \\ Taiwan \\ ${ }^{4}$ Institute of Electro-optical Science and Technology, National Taiwan Normal University, \\ Taipei 106, Taiwan
}

E-mail: hcyang@phys.ntu.edu.tw (H-C Yang)

Received 24 October 2005, in final form 7 December 2005

Published 27 February 2006

Online at stacks.iop.org/SUST/19/S246

\begin{abstract}
We have designed and fabricated a low noise, high $T_{\mathrm{c}}$ superconducting quantum interference device (SQUID) magnetometer with an improved yield. In order to reduce the field noise level of the magnetometer, we increased the voltage modulation depth with a series SQUID array and enhanced the effective area with a flux focuser. Ten washer-type SQUIDs were connected in series, thereby increasing the voltage flux transfer function by a factor of 10. An enhancement in effective area by a factor of 5 was achieved by coupling the 10-SQUID array to a single-layered flux focuser. For the directly coupled SQUID array magnetometer, the yield was improved by selecting the best SQUIDs to have in series in order to reduce the flux noise. The magnetic field sensitivity of $40 \mathrm{fT} \mathrm{Hz}^{-1 / 2}$ in the white regime and $100 \mathrm{fT} \mathrm{Hz}^{-1 / 2}$ at $1 \mathrm{~Hz}$ is demonstrated by using a single layer of high $T_{\mathrm{c}}$ film. The proposed high yield magnetometers would be suitable for building low noise multi-channel magnetocardiographs.
\end{abstract}

(Some figures in this article are in colour only in the electronic version)

\section{Introduction}

The superconducting quantum interference device (SQUID) is the most sensitive device for detecting a weak magnetic flux. SQUID magnetometers have unsurpassed energy sensitivity and have been used to provide images of the magnetic field distributions associated with nerve and muscle currents, currents in integrated circuits, trapped flux in superconductors and defects in metals, and Johnson noise [1]. Active research in the development of high $T_{\mathrm{c}}$ SQUIDs is being conducted worldwide and commercialization is expected soon. The lower flux noise and large output voltage are very important for the above applications. According to the sensitivity relation $S_{B}^{1 / 2}(f)=S_{\Phi}^{1 / 2}(f) / A_{\text {eff }}$, it is crucial to reduce the flux noise and have a large effective area for HTS sensitive SQUIDs [1-3]. Many studies have so far been made for

\footnotetext{
5 Author to whom any correspondence should be addressed.
}

the development of highly sensitive SQUID magnetometers operating at $77 \mathrm{~K}$. With low $T_{\mathrm{c}}$ technology, it is shown that by using serially connected SQUID arrays the flux-tovoltage transfer function can be improved and the flux noise reduced [4]. Low flux noise is attractive for magnetometer applications. However, for a serial SQUID magnetometer the reduction in flux noise would be accompanied by a reduction in effective area. Moreover, the non-negligible spread of junction parameters and non-uniformity of magnetic flux density in the array may further degrade the field noise level. Owing to trouble with the SQUID array, high sensitivity, low $T_{\mathrm{c}}$ SQUID magnetometers with multiple turns reported to date were realized by enhancing the effective area rather than reducing the flux noise with a SQUID array. With high $T_{\mathrm{c}}$ technology, a single-layered device is preferred owing to the difficulty in engineering multi-layered high $T_{\mathrm{c}}$ film. For a single-layered directly coupled magnetometer $[5,6]$, the effective area is typically $0.2 \mathrm{~mm}^{2}$ and the typical field noise level is about 
$100 \mathrm{fT} \mathrm{Hz}^{-1 / 2}$ on a $10 \times 10 \mathrm{~mm}^{2}$ chip [7]. It seems possible to further reduce the field noise by using a high $T_{\mathrm{c}}$ serial SQUID array. However, for the works reported to date [8-10] the performances of magnetometers in serial arrays are limited by the small effective area and the spread of the junction parameters. Briefly, some other techniques must be applied to exploit the advantages of the serial SQUID array for low noise magnetometers.

In this work, we investigate a magnetometer consisting of a washer-type SQUID array to obtain a large effective area and reduce the white flux noise. The serial SQUID array increases the voltage modulation depth while the washer-type loop with a flip-chip flux focuser enhances the effective area [11-14]. The optimal performance achievable with the high $T_{\mathrm{c}}$ serial SQUID array with a flip-chip flux focuser is discussed.

\section{Experimental details}

In figure 1(a), we show the layout of our SQUID magnetometer with a flip-chip flux transformer. The width of the junction is $3 \mu \mathrm{m}$. The area of the SQUID hole was $30 \times 30 \mu \mathrm{m}^{2}$. The calculated geometrical inductance of the SQUID loop is $38 \mathrm{pH}$. Ten SQUIDs in series were aligned along a bi-crystal line $5 \mathrm{~mm}$ long, shown in figure 1(b). The serial SQUIDs were fabricated onto $5 \mathrm{~mm} \times 5 \mathrm{~mm} \mathrm{SrTiO}_{3}$ bi-crystal substrates with $36.8^{\circ}$ misorientation angle. The film thickness is $150 \mathrm{~nm}$. The superconducting $\mathrm{YBa}_{2} \mathrm{Cu}_{3} \mathrm{O}_{7}$ (YBCO) films were grown with high oxygen pressure off-axis pulsed laser ablation [15-17]. High $T_{\mathrm{c}}$ YBCO serial SQUIDs were fabricated using the standard techniques of photolithography and $\mathrm{Ar}^{+}$ion milling. Gold contacts are evaporated at about $300 \mathrm{~nm}$ thickness from the SQUID to the back of the substrate. In the flip-chip configuration, the SQUID is fabricated on one substrate and the flux focuser is fabricated on another substrate. The input coil of the transformer is aligned with the SQUID loop. In the design of the thin film flux focuser, $20 \times 20 \mathrm{~mm}^{2}$, shown in figure 1(a), the flux over a larger area is concentrated on the small hole of $5 \times 3 \mathrm{~mm}^{2}$ at the centre. Thus, a much larger flux is coupled to the SQUID, leading to an enhancement in the magnetic field sensitivity of the SQUID. The voltage versus current $(V-I)$ and voltage versus flux $(V-\Phi)$ characteristics of magnetometers were measured using NKT's DC SQUID Catcher ${ }^{\mathrm{TM}}$ system. The flux noise spectra of the magnetometers were measured by using Conductus's multi-channel PC interface, PC-1000, with programmable feedback loops, PFL-100.

\section{Results and discussion}

The SQUID is generally operated on the steep part of the $V-\Phi$ curve where the transfer coefficient, $V_{\Phi}$, is a maximum. Thus, the SQUID produces an output voltage in response to a small input flux $\delta \Phi$, and is effectively a flux-to-voltage transducer. The relatively small voltage signal of a single SQUID can be enlarged considerably by connecting many identical SQUIDs in series. Provided that all have the same working point and that the same flux $\Phi$ is coupled into each of the SQUIDs, the array behaves like a single SQUID with an enhanced voltage swing. The flux noise spectral density $S_{\Phi}(f)$ in a high $T_{\mathrm{c}}$ series SQUID array can be derived from known formulae for voltage (a)

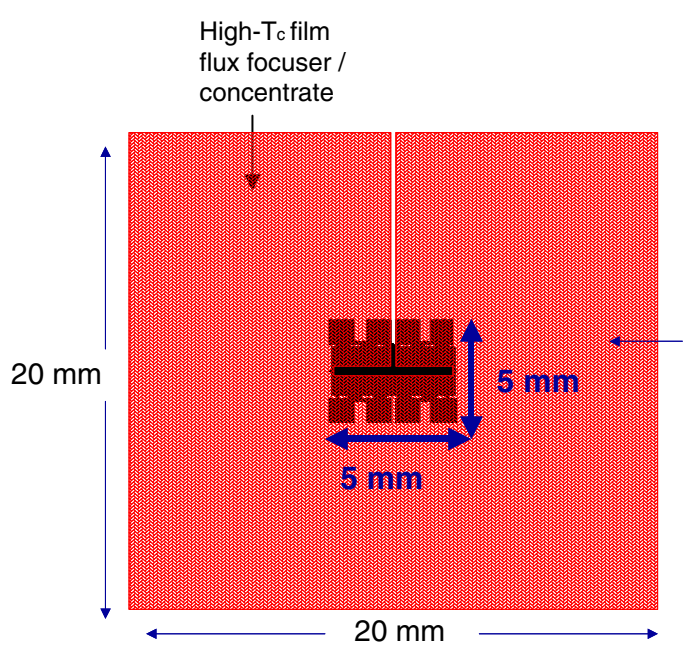

(b)

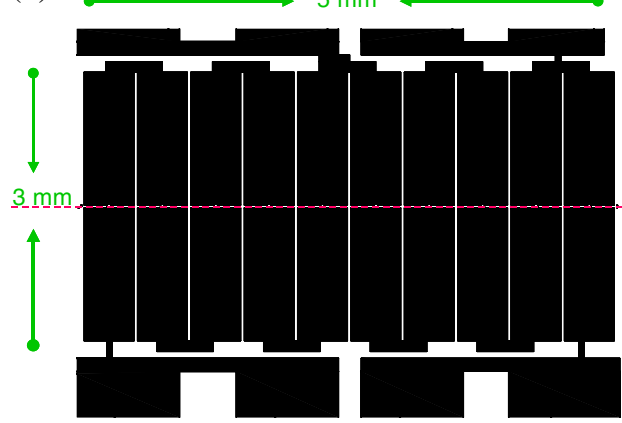

(c)

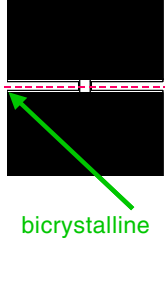

Figure 1. (a) Schematic diagram of the flip-chip high $T_{\mathrm{c}}$ SQUID magnetometer. (b) A 10-SQUID serial array was aligned along a bi-crystal line. (c) The junction width is $3 \mu \mathrm{m}$. The distance between two SQUIDs was $15 \mu \mathrm{m}$. The hole area of the SQUID was $30 \mu \mathrm{m}^{2}$.

noise and voltage swing of a single SQUID. Given the voltage noise power spectral density of a SQUID being $S_{\mathrm{V}}(f)$, the flux noise power spectral density, $S_{\Phi}(f)$, for a single SQUID is given by

$$
S_{\Phi}(f)=\frac{S_{\mathrm{V}}(f)}{\left(\frac{\partial V}{\partial \Phi}\right)^{2}} .
$$

Enpuku et al [18] concludes empirical formulae for the voltage modulation depth $\Delta V$ and flux-to-voltage transfer function $V_{\Phi}$ of a single SQUID to be

$$
\begin{gathered}
\Delta V=\frac{7}{\pi^{2}} \frac{I_{\mathrm{c}} R_{\mathrm{n}}}{\left(1+\beta_{L}\right)}\left(1-3.57 \frac{\sqrt{k_{\mathrm{B}} T L}}{\Phi_{0}}\right), \\
\text { with } \quad V_{\Phi}=\frac{\partial V}{\partial \Phi} \cong \pi \frac{\Delta V}{\Phi_{0}}
\end{gathered}
$$

where $I_{\mathrm{c}}$ and $R$ are the critical current and normal resistance of a Josephson junction, $L$ is the SQUID loop inductance, $T$ is the SQUID operating temperature, $k_{\mathrm{B}}$ is Boltzmann's constant, and $\Phi_{0}$ is the fluxon. If $N$ SQUIDs are connected serially in an array and when all SQUID output voltages add coherently, the total array voltage modulation depth is

$$
\begin{gathered}
(\Delta V)_{\text {array }}=N \cdot(\Delta V)_{\text {SQUID }}, \quad\left(V_{\Phi}\right)_{\text {array }}=N \cdot\left(V_{\Phi}\right), \\
S_{\mathrm{V}}(f)_{\text {array }}=N \cdot S_{\mathrm{V}}(f) .
\end{gathered}
$$




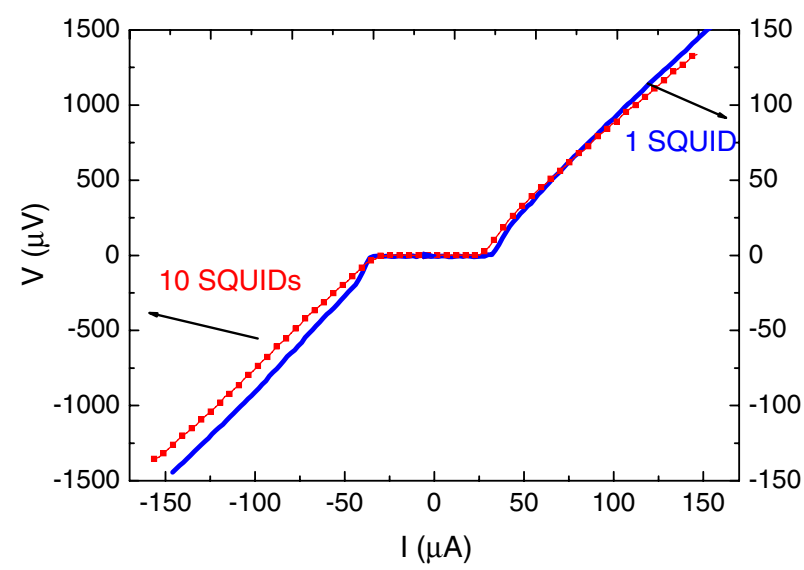

Figure 2. $I-V$ characteristics of a single SQUID and 10 SQUIDs connected in series at $77 \mathrm{~K}$.

By using equations (1)-(3) we see that the flux noise spectral density is $S_{\Phi}(f)_{\text {array }}=S_{\Phi}(f) / N$, decreasing with $1 / \sqrt{ } N$ where $N$ is the number of SQUIDs [1, 19]. For ten SQUIDs connected in series with all the SQUID output voltages added coherently, the white flux noise should decrease to $1 / \sqrt{ } 10$ the noise of a single SQUID.

Figure 2 shows the typical $I-V$ characteristics of a singleSQUID magnetometer at $77 \mathrm{~K}$. The current-voltage curves fit well with the resistively shunted junction (RSJ) model under thermally activated phase slippage [20]. According to the fitting with the RSJ model under thermal noise, the SQUID has the normal resistance of $1.1 \Omega$ and critical current of $37 \mu \mathrm{A}$, giving the characteristic voltage of $I_{\mathrm{c}} R_{\mathrm{n}}=41 \mu \mathrm{V}$ at $77 \mathrm{~K}$. Figure 2 also shows $I-V$ characteristics for 10 SQUIDs connected in series. The normal state resistance was found to increase when the number of SQUIDs was increased. By adapting the same single-junction RSJ model with therma noise to the SQUID array, the normal state resistance is found to be $9.8 \Omega$ and the critical current to be $33 \mu \mathrm{A}$. The resistance does scale with the number of the SQUIDs in series. This implies that the spread in normal resistance of the SQUID is small. In comparison with the critical current of a single SQUID, the slight reduction in the critical current of the array suggests that the spread in critical currents may be small for this sample. In figure 3(a), we show the voltage flux characteristic curve, $V-I_{\text {mod }}$, of the single SQUID and the 10SQUID array at $77 \mathrm{~K}$. The peak-to-peak voltage swing is about $3.2 \mu \mathrm{V}$ for a single SQUID. The small flux-to-voltage transfer function resulted in a small slew rate and made it difficult to lock the SQUID with the flux modulation scheme by using the commercially available programmable feedback loop PFL100 .

Although the voltage swing of one SQUID is small on this chip, the voltage swing is enhanced effectively with the 10-SQUID series array as shown in figure 3(a). With the uniform flux focusing of the washer, the voltage flux curves of the SQUIDs in the array were superimposed coherently. The voltage modulation depth is increased from $3.2 \mu \mathrm{V}$ for one SQUID to $30 \mu \mathrm{V}$ for the 10-SQUID array. The peak-topeak voltage swing of the 10-SQUID magnetometer is roughly tenfold compared with that of the one-SQUID magnetometer. In figure 3(b), the voltage flux curves for the 10-SQUID array
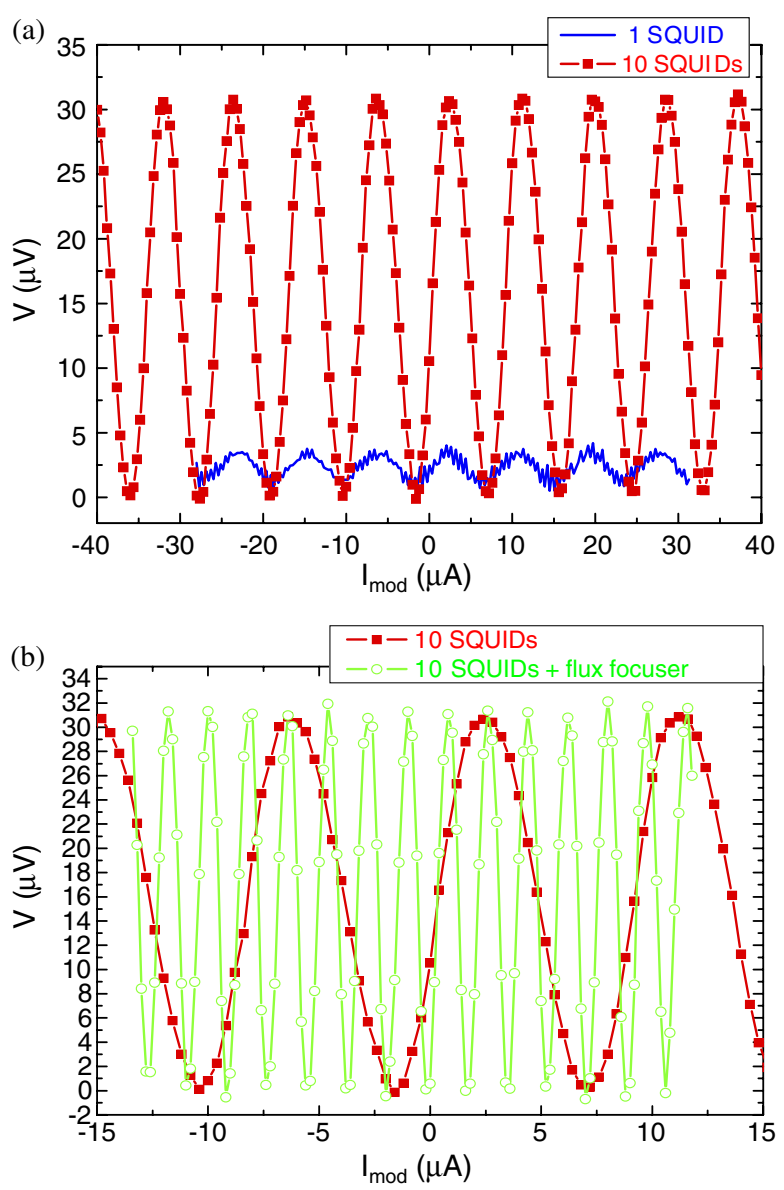

Figure 3. (a) Modulation current-voltage curve of a single SQUID and 10 serial SQUIDs. (b) $I_{\bmod }-V$ curve of 10 serial SQUIDs with a flux focuser and without it.

with and without a flux focuser are shown. The smaller period for the case with the flux focuser corresponds to a larger effective area. The effective area without the flux focuser is $0.037 \mathrm{~mm}^{2}$ while that with the flux focuser is $0.185 \mathrm{~mm}^{2}$. The increase in effective area reveals the effectiveness of the flux focuser. As the beating phenomenon is not obvious in the voltage flux curve with the flux focuser, the magnetic flux density at the flux focuser is quite uniform. Hence, by using the washer SQUID with the flux focuser, the effective area can be enhanced effectively by a factor of about 5 .

Figure 4 shows the corresponding magnetic field sensitivity for 10 SQUIDs without and with a flux focuser. Without a flux focuser, the 10-SQUID magnetometer showed the magnetic field sensitivity of about $600 \mathrm{fT} \mathrm{Hz}^{-1 / 2}$ at $1 \mathrm{kHz}$ and $1.5 \mathrm{pT} \mathrm{Hz}^{-1 / 2}$ at $1 \mathrm{~Hz}$ in a shielded environment. With the flux focuser, the magnetic field noise level of the 10-SQUID flip-chip magnetometer was improved to $150 \mathrm{fT} \mathrm{Hz}^{-1 / 2}$ at $1 \mathrm{kHz}$ and $400 \mathrm{fT} \mathrm{Hz}^{-1 / 2}$ at $1 \mathrm{~Hz}$. The reduction in field noise level was caused by the increase in effective area with the flux focuser. However, we note that the field noise is reduced by a factor of 4 rather than 5 as expected from the enhancement in effective area. The slight discrepancy was not caused by the flux motion in flux focuser since there was no strong increase in low frequency flux noise. By investigating the voltage flux curves carefully, we found that the presence of the flux focuser 
A highly sensitive YBCO serial SQUID magnetometer with a flux focuser

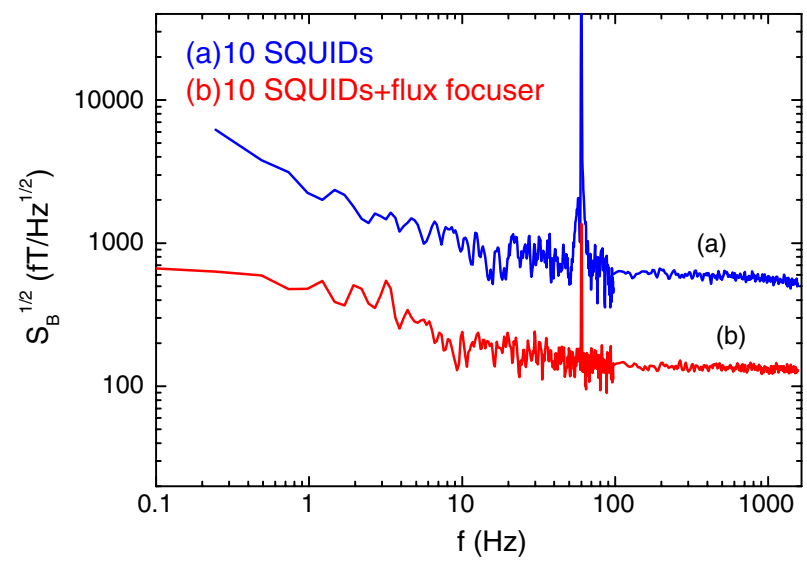

Figure 4. The magnetic field sensitivity of (a) the serial SQUID magnetometer, (b) SQUIDs with flux focuser.

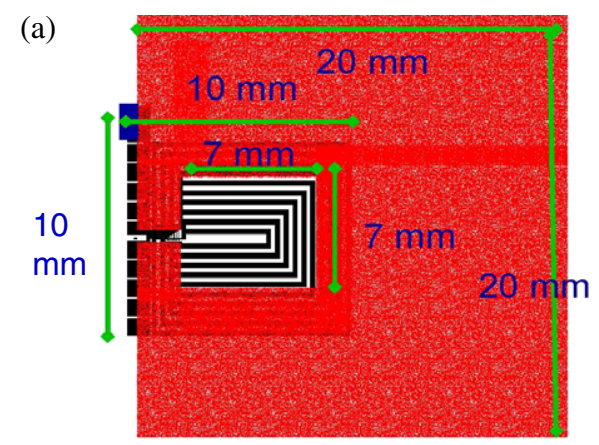

(b)

(c)

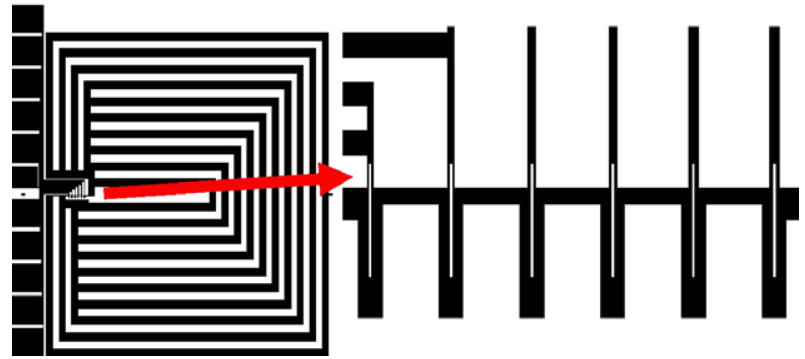

Figure 5. (a) Schematic diagram of a directly coupled SQUID with a flux focuser. (b) A six-SQUID array was aligned along a bi-crystal line. (c) The junction width is $3 \mu \mathrm{m}$. The distance between two SQUIDs was $15 \mu \mathrm{m}$. The SQUID inductance is $65 \mathrm{pH}$.

altered the 'shape' of the voltage flux characteristic curves. Namely, the deviation from the sinusoidal approximation in (2) differs for the voltage flux curves with and without a flux focuser.

Although the serial SQUID array demonstrated above shows a reduction in flux noise, the yield of the 10-SQUID array is not high since the on-chip critical spread of bi-crystal Josephson junctions is usually not small at $77 \mathrm{~K}[21,22]$. To improve the yield of a SQUID array magnetometer with bi-crystal Josephson junctions, the best way would be to fabricate many SQUIDs and choose the best ones for being connected in series. To do this, we designed and fabricated the directly coupled SQUID magnetometer for series array operation shown in figure 5. The SQUID magnetometer

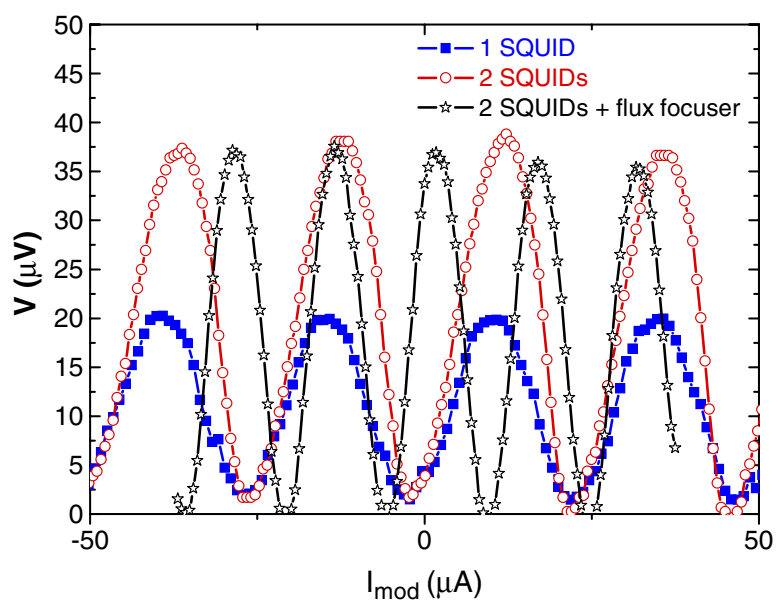

Figure 6. $I_{\bmod }-V$ curve of a single SQUID, two serial SQUIDs and two SQUIDs with a flux focuser.

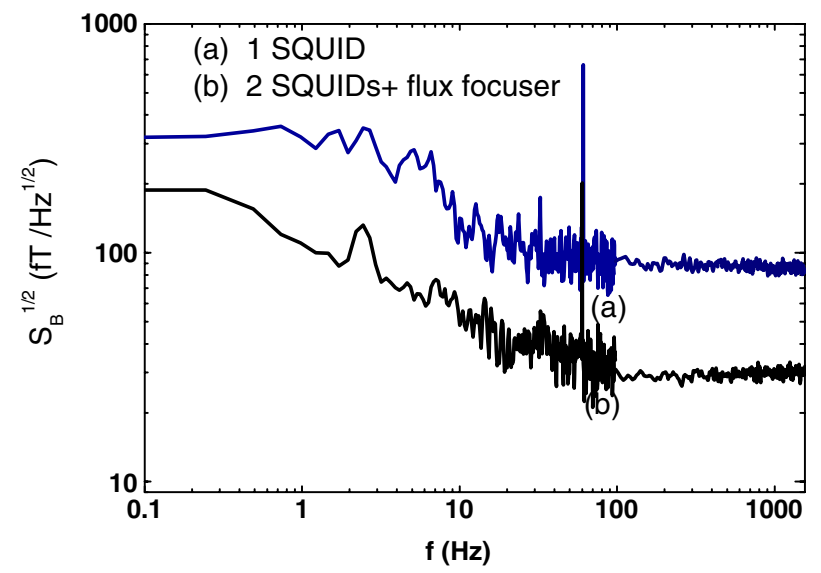

Figure 7. The magnetic field sensitivity of (a) a one-SQUID magnetometer, (b) two SQUIDs with a flux focuser.

was fabricated onto a $10 \mathrm{~mm} \times 10 \mathrm{~mm} \mathrm{SrTiO}_{3}$ bi-crystal substrate with a $24^{\circ}$ misorientation angle. The junction width is $3 \mu \mathrm{m}$ and the SQUID inductance is $65 \mathrm{pH}$. The bare SQUIDs share a common pick-up loop to form the directly coupled SQUID magnetometer. To further reduce the flux noise with the SQUID array, any two out of the six bare SQUIDs can be connected in series for the directly coupled magnetometer. Figure 6 shows the $V-\Phi$ curve of the directly coupled magnetometer. The voltage swing was $19 \mu \mathrm{V}$ for the single-SQUID magnetometer and $37 \mu \mathrm{V}$ for the two-SQUID series array magnetometer. The amplification in voltage swing by a factor of 2 would lead to a reduction in white flux noise by a factor of 1.4 according to equation (3). By measuring the periods of the voltage flux curves in figure 6 , the effective areas were determined as $0.2 \mathrm{~mm}^{2}$ for both the single-SQUID and two-SQUID array magnetometers without the flux focuser. When the flip-chip flux focuser was applied, the effective areas were boosted by a factor of 1.6 to $0.32 \mathrm{~mm}^{2}$. Therefore, by using the two-SQUID array with a flip-chip flux focuser, the field sensitivity can be improved by a factor of $1.4 \times 1.6=2.24$. Figure 7 shows the corresponding magnetic field noise spectral densities respectively for the single-SQUID magnetometer without a flux focuser and the 
two-SQUID-array magnetometer with a flux focuser. For the conventional single-SQUID magnetometer without a flux focuser, the magnetic field noise level was $90 \mathrm{fT} \mathrm{Hz}^{-1 / 2}$ at $1 \mathrm{kHz}$ and $350 \mathrm{fT} \mathrm{Hz}^{-1 / 2}$ at $1 \mathrm{~Hz}$ in a shielded environment. For the two-SQUID-array magnetometer with flux focuser, the magnetic field noise level was reduced to $40 \mathrm{fT} \mathrm{Hz}^{-1 / 2}$ at $1 \mathrm{kHz}$ and $120 \mathrm{fT} \mathrm{Hz}^{-1 / 2}$ at $1 \mathrm{~Hz}$. The observed reduction in white field noise level by a factor of 2.25 was in agreement with the observed amplifications in voltage swing and effect area. Since the flux focuser and SQUID array are made from single-layered high $T_{\mathrm{c}}$ thin films on separate chips, the yield of our low noise magnetometer should be much higher than that of high $T_{\mathrm{c}}$ SQUID magnetometers with multi-layered flux transformers [23].

\section{Conclusion}

We have characterized a high $T_{\mathrm{c}}$ YBCO dc SQUID array magnetometer fabricated on a bi-crystal STO substrate. The improvement in magnetic field sensitivity $S_{B}^{1 / 2}$ was achieved by reducing the flux noise $S_{\Phi}^{1 / 2}$ with a SQUID array and enhancing the effective area $A_{\text {eff }}$ with a flip-chip flux focuser. For the washer-type 10-SQUID array, the enhancement in the effective area by a factor of 5 was achieved with a singlelayered flux focuser. By using single-layered YBCO thin films, a high $T_{\mathrm{c}}$ directly coupled SQUID magnetometer with a flux focuser exhibiting a magnetic field sensitivity of $40 \mathrm{fT} \mathrm{Hz}^{-1 / 2}$ in the white regime and $100 \mathrm{fT} \mathrm{Hz}^{-1 / 2}$ at $1 \mathrm{~Hz}$ was achieved. Our design can improve the yield and reduce the flux noise by selecting the best SQUIDs for operation in a serial array. From the standpoint of application, it is quite practicable to build a high sensitivity multi-channel SQUID system for magnetocardiograms by using the proposed magnetometers.

\section{Acknowledgments}

The authors are grateful for the financial support of the Ministry of Education (91-N-FA01-2-4-2) and the National Science Council of Taiwan (NSC94-2112-M002-015).

\section{References}

[1] Braginski A I 1995 Fabrication and Applications ed H Weinstock (Dordrecht: Kluwer-Academic) p 235

[2] Welty R P et al 1991 IEEE. Trans. Magn. 272924

[3] Welty R P et al 1993 IEEE. Trans. Appl. Supercond. 3 2605-9

[4] Stawiasz K G and Ketchen M B 1993 IEEE Trans. Appl. Supercond. 31808

[5] Koelle D, Miklich A H, Ludwig F, Dantsker E, Nemeth D T and Clarke J 1993 Appl. Phys. Lett. 632271

[6] Matsuda M, Nakamura K, Mikami H and Kuriki S 2005 IEEE Trans. Appl. Supercond. 15817

[7] Cantor R 1996 SQUID Sensors: 1996 Fundamentals, Fabrication and Applications (NATO ASI Series) ed H Weinstock (Dordrecht: Kluwer-Academic) p 179

[8] Lee S G, Huh Y, Park G S, Kim I S, Park Y K and Park J C 1997 IEEE Trans. Appl. Supercond. 73347

[9] Schultze V, Zakosarenko V, Usselsteijn R, Ramos J and Meyer H G 1999 IEEE Trans. Appl. Supercond. 93279

[10] Wu C H, Chen M J, Hsu M H, Chen J H, Chen K L, Chen J C, Lai T S, Horng H E and Yang H C 2005 Physica C 433 108-14

[11] Faley M I, Poppe U, Urban K, Paulson D N, Starr T N and Fagaly R L 2001 IEEE Trans. Appl. Supercond. 11 1383-6

[12] Ramos J, Chwala A, Ijsselsteijn R, Stolz R, Zakosarenko V, Schultze V, Hoenig H E, Meyer H-G, Beyer J and Drung D 1999 IEEE Trans. Appl. Supercond. $93392-5$

[13] Zhang Y 1997 Appl. Phys. Lett. 71 704-6

[14] Borgmann J 1997 Rev. Sci. Instrum. 68 2730-4

[15] Chen K L, Chen J H, Horng H E and Yang H C 2002 Physica C 3721078

[16] Wu C H, Chen J H, Chen K L, Chen J C, Horng H E and Yang H C 2005 Chin. J. Phys. 43675

[17] Yang H C, Chen J H, Chen K L, Chen M J, Wu C H, Jeng J T and Horng H E 2002 IEICE Trans. Electron. 85772

[18] Enpuku K, Shimomura Y and Kisu T 1993 J. Appl. Phys. 737929

[19] Neeraj Khare 2003 Handbook of High-Temperature Superconductor Electronics (New York, USA: Marcel Dekker)

[20] Ambegaokar V and Halperin B I 1969 Phys. Rev. Lett. 221364

[21] Shadrin P, Jia C L and Divin Y 2003 IEEE Trans. Appl. Supercond. 13603

[22] Jeng J T, Hung H C, Lin C R, Wu C H, Chen K L, Chen J C, Yang H C, Liao S H and Horng H E 2005 IEEE Trans. Appl. Supercond. 15793

[23] Faley M I, Poppe U, Urban K, Paulson D N, Starr T N and Fagaly R L 2001 IEEE Trans. Appl. Supercond. 111383 